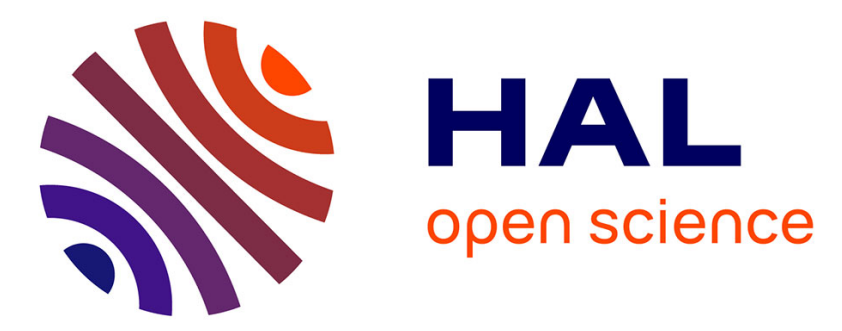

\title{
Utilisation de la typologie automatique pour la mise au point d'un échantillonnage stratifié, en vue de l'étude des relations plante hôte-pucerons en verger de pêchers
}

\author{
Laurent Lapchin, Roger Boll
}

\section{- To cite this version:}

Laurent Lapchin, Roger Boll. Utilisation de la typologie automatique pour la mise au point d'un échantillonnage stratifié, en vue de l'étude des relations plante hôte-pucerons en verger de pêchers. Agronomie, 1981, 1 (7), pp.587-594. hal-00884299

\section{HAL Id: hal-00884299 \\ https://hal.science/hal-00884299}

Submitted on 1 Jan 1981

HAL is a multi-disciplinary open access archive for the deposit and dissemination of scientific research documents, whether they are published or not. The documents may come from teaching and research institutions in France or abroad, or from public or private research centers.
L'archive ouverte pluridisciplinaire HAL, est destinée au dépôt et à la diffusion de documents scientifiques de niveau recherche, publiés ou non, émanant des établissements d'enseignement et de recherche français ou étrangers, des laboratoires publics ou privés. 


\title{
Utilisation de la typologie automatique pour la mise au point d'un échantillonnage stratifié, en vue de l'étude des relations plante hôte-pucerons en verger de pêchers
}

Laurent LAPCHIN \& Roger BOLL

I.N.R.A., Station de Zoologie et de Lutte biologique, 37, Bd du Cap, F 06602 Antibes

RÉSUMÉ

Pêchers,

Aphides,

Echantillonnage

stratifié,

Phénologie,

Typologie automatique.

\begin{abstract}
Dans un verger de jeunes pêchers, destinés à l'étude de leurs relations avec les populations de pucerons, le choix des arbres-échantillons est basé sur des variables phénologiques : diamètre du tronc ; nombre, longueur et degré de ramification des pousses de l'année ; précocité du développement des feuilles au printemps et de leur jaunissement à l'automne.

L'analyse factorielle des correspondances met en évidence l'évolution parallèle de plusieurs variables ; diamètre du tronc, longueur et ramification des pousses évoluent en sens inverse de la précocité du développement foliaire et du nombre de rameaux.

La classification ascendante hiérarchique est appliquée aux coordonnées des arbres sur les axes factoriels. Le calcul d'un indice est proposé pour faciliter le choix d'un niveau de coupure de l'arbre hiérarchique. Onze groupes sont finalement conservés, dont les profils phénologiques sont définis à l'aide des indices d'association et de signification de BORDET \& Albuisson (1974). Cinq arbres par groupe sont enfin choisis de façon à recouvrir le plus uniformément possible la surface du verger.
\end{abstract} interactions in peach-tree orchards

Peach trees, Aphids, Stratified sampling, Phenology, Cluster analysis.

\begin{abstract}
A young peach-tree orchard at Fréjus (South-eastern part of France) is studied with regards to aphid populations. In order to determine sampling trees, phenological data are collected : stem diameter, number, length and ramifying ratio of shoots, precocity of spring foliar development and autumn defoliation.

As pointed out with correspondance analysis, stem diameter, shoot length and ramifying ratio have variations reverse to shoot number and precocity of spring foliar development.

A hierarchical ascendant classification is made from coordinates of trees on factorial axes. An index is proposed to help in determination of the hierarchical tree cutting level. Eleven groups are obtained. Their phenological patterns are defined with association and signification indexes by BORDET \& ALBUISSON (1974). Five trees per group are finally determined in order to obtain a spatial distribution as regular as possible.
\end{abstract}

\section{INTRODUCTION}

L'étude des relations entre la plante hôte et les aphides, en verger de Rosacées, concerne aussi bien l'impact du phytophage sur la physiologie et la phénologie de l'arbre que l'influence de celui-ci sur l'installation et le développement des populations de ravageurs. De nombreux paramètres du milieu (climat, microclimat, cultures environnantes, sol, techniques culturales, etc.) interfèrent par ailleurs avec ces relations. L'échantillonnage des espèces aphidiennes en verger doit donc tenir compte de l'ensemble de ces éléments.

Le choix du nombre de plantes à examiner nécessite un compromis entre la quantité d'information souhaitée, en fonction de la précision attendue des résultats, et le temps disponible pour recueillir cette information. Dans cet esprit, la stratification de la population d'arbres étudiés permet de rentabiliser au maximum les données collectées et s'avère préférable à un simple tirage aléatoire.

Dans le cas d'un verger de Rosacées fruitières, monovariétal et d'âge homogène, les caractéristiques phénologiques des arbres peuvent constituer une bonne représentation de leur individualité. Certains paramètres de cette phénologie sont par ailleurs assez rapides et faciles à collecter. Une étude préliminaire a donc été effectuée dans un jeune verger de pêchers destiné à l'examen des relations arbrespucerons, de manière à déterminer une cinquantaine d'individus échantillons. 


\section{LIEU D'ÉTUDE, TECHNIQUES ET MÉTHODES}

Le verger expérimental est situé dans le domaine de la Station d'Amélioration des Plantes de Fréjus (Var), au confluent des plaines alluviales de l'Argens et du Reyran. Il comporte 132 pêchers (cv. "Redhaven ", greffés sur franc) pour une superficie de $2200 \mathrm{~m}^{2}$. Ces arbres ont été plantés en février 1980 , avec un espacement de $4 \times 5 \mathrm{~m}$. La parcelle prolonge un verger de même variété, âgé de 12 ans, situé au nord ; bordée à l'est et à l'ouest de brise-vent de cyprès, elle côtoie au sud des cultures mixtes de vignes et de pêchers. Afin de permettre l'étude du développement des ravageurs, les interventions chimiques ont été limitées à l'application d'un traitement cuprique peu après la plantation.

Les paramètres phénologiques ont été examinés de l'hiver à l'automne 1980. Il s'agit de variables qualitatives ou quantitatives: le diamètre du tronc à $1 \mathrm{~m}$ du sol rend compte de la puissance de l'arbre au moment de la plantation; les longueurs moyennes des feuilles au début et à la fin du mois de mars représentent la précocité du développement végétatif ; en juillet, le nombre de rameaux de l'année, leur degré de ramification et la longueur du plus grand d'entre eux décrivent les caractéristiques de la pousse de bois de la saison; enfin, la précocité du jaunissement des feuilles a été appréciée à la fin du mois d'octobre.

L'ensemble de ces données a été partagé en classes, à partir des histogrammes de distribution de fréquence pour les variables quantitatives ou des indices des variables qualitatives, pour produire un codage disjonctif complet. Le tableau 1 présente les différents paramètres et leur codage. Sept arbres, morts à la fin de l'expérience, ont été exclus du dépouillement. Le tableau comportant les 23 modalités descriptives des 125 arbres restants a été soumis à l'analyse factorielle des correspondances. Une classification ascendante hiérarchique, basée sur le critère de la maximisation du moment centré d'ordre 2 , a ensuite été réalisée d'après les coordonnées des arbres sur les axes factoriels, suivant la méthode décrite par JAMBU (1978) et JAMBU \& LEBEAUX (1978). Les traitements informatiques ont été effectués sur le matériel calcul du Centre de Recherches agronomiques d'Antibes.

\section{RÉSULTATS}

\section{A. Analyse factorielle des correspondances}

L'analyse factorielle des correspondances dégage 16 valeurs propres non triviales et non nulles, associées à 16 axes factoriels. Les pourcentages de l'inertie totale représentés par les axes successifs sont assez régulièrement décroissants, exprimant une structure relativement complexe (fig. 1) ; la complexité apparente est d'ailleurs accentuée par le codage disjonctif complet qui multiplie le nombre d'axes factoriels. Compte tenu de la légère rupture de pente observée, sur l'histogramme des pourcentages d'inertie, entre les axes 4 et 5 , les 4 premiers axes ont été conservés pour l'interprétation de l'analyse et la réalisation de la classification ascendante hiérarchique.

L'axe 1 représente 16 p. 100 de l'information totale et ordonne les modalités de toutes les variables, hormis le jaunissement des feuilles. Le diamètre du tronc, la longueur et le degré de ramification des rameaux évoluent en sens inverse du développement foliaire et du nombre de rameaux principaux. Trois modalités sont décrites essentiellement par cet axe (contributions relatives de l'ordre de 50 p. 100) :
TABLEAU 1

Codage et dates de relevé des différentes variables Codage and sampling dates of data

\begin{tabular}{|c|c|c|c|c|}
\hline Variables & Dates & Codes & $\begin{array}{c}\text { Limites } \\
\text { des classes }\end{array}$ & Effectifs \\
\hline \multirow[t]{4}{*}{$\begin{array}{l}\text { Diamètre du tronc à } \\
1 \mathrm{~m} \text { du sol }\end{array}$} & $14-02-80$ & DT1 & $7-11 \mathrm{~mm}$ & 23 \\
\hline & & DT2 & $12-13$ & 37 \\
\hline & & DT3 & $14-15$ & 41 \\
\hline & & DT4 & $16-18$ & 24 \\
\hline \multirow[t]{3}{*}{$\begin{array}{l}\text { Longueur du plus } \\
\text { grand rameau }\end{array}$} & $02-07-80$ & LM1 & $5-40 \mathrm{~cm}$ & 29 \\
\hline & & LM2 & $41-60$ & 61 \\
\hline & & LM3 & $61-85$ & 35 \\
\hline \multirow[t]{3}{*}{$\begin{array}{l}\text { Nombre de rameaux } \\
\text { principaux }\end{array}$} & $02-07-80$ & NR1 & $1-4$ & 40 \\
\hline & & NR2 & $5-7$ & 58 \\
\hline & & NR3 & $8-12$ & 27 \\
\hline \multirow[t]{3}{*}{$\begin{array}{l}\text { Degré de ramifica- } \\
\text { tion secondaire }\end{array}$} & $02-07-80$ & RA1 & faible ou nul & 48 \\
\hline & & RA2 & moyen & 35 \\
\hline & & RA3 & fort & 42 \\
\hline \multirow[t]{3}{*}{$\begin{array}{l}\text { Longueur moyenne } \\
\text { des feuilles }\end{array}$} & $04-03-80$ & F11 & $0-3 \mathrm{~mm}$ & 35 \\
\hline & & $\mathrm{F} 12$ & $3-5$ & 65 \\
\hline & & F13 & $>5$ & 25 \\
\hline \multirow[t]{4}{*}{$\begin{array}{l}\text { Longueur moyenne } \\
\text { des feuilles }\end{array}$} & $17-03-80$ & $\mathrm{~F} 21$ & $0-3 \mathrm{~mm}$ & 88 \\
\hline & & $\mathrm{F} 22$ & $4-14$ & 49 \\
\hline & & $\mathrm{F} 23$ & $15-24$ & 50 \\
\hline & & $\mathrm{F} 24$ & $25-40$ & 18 \\
\hline \multirow[t]{3}{*}{$\begin{array}{l}\text { Degré de jaunisse- } \\
\text { ment des feuilles }\end{array}$} & $22-10-80$ & CF1 & vert & 60 \\
\hline & & $\mathrm{CF} 2$ & vert - jaune & 58 \\
\hline & & $\mathrm{CF} 3$ & jaune & 7 \\
\hline
\end{tabular}

NR 1, RA 1 et RA 3. Sept des modalités définissent à elles seules 72 p. 100 de l'axe (contributions absolues) : il s'agit de l'association F 11, NR 1, RA 3 (rameaux de l'année peu nombreux et ramifiés; feuillage tardif), opposée au groupe F 13, F 24, NR 3, RA 1 (rameaux de l'année nombreux et sans ramification; feuillage précoce). Le premier ensemble est plutôt lié aux forts diamètres du tronc et aux grandes longueurs de rameaux (DT 4, LM 3), le second aux faibles diamètres, voire aux faibles longueurs de rameaux (DT 1, LM 1).

L'axe 2 (12 p. 100 de l'information totale) représente, sur sa partie positive, des arbres à rameaux courts (LM 1) qui développent toutefois un feuillage tardif (F 11, F 21). Ces individus présentent souvent un nombre de rameaux moyen (NR 2) et un jaunissement des feuilles également tardif (CF 1). Le diamètre du tronc est rarement très fort. La partie négative de l'axe regroupe les arbres à tronc puissant (DT 4) et à rameaux longs (LM 3), renforçant l'association observée sur l'axe 1, mais auxquels sont souvent liés un feuillage assez développé à la mi-mars (F 23) et une précocité moyenne du jaunissement des feuilles (CF 2). L'ensemble de ces modalités détermine 77 p. 100 du second axe. 


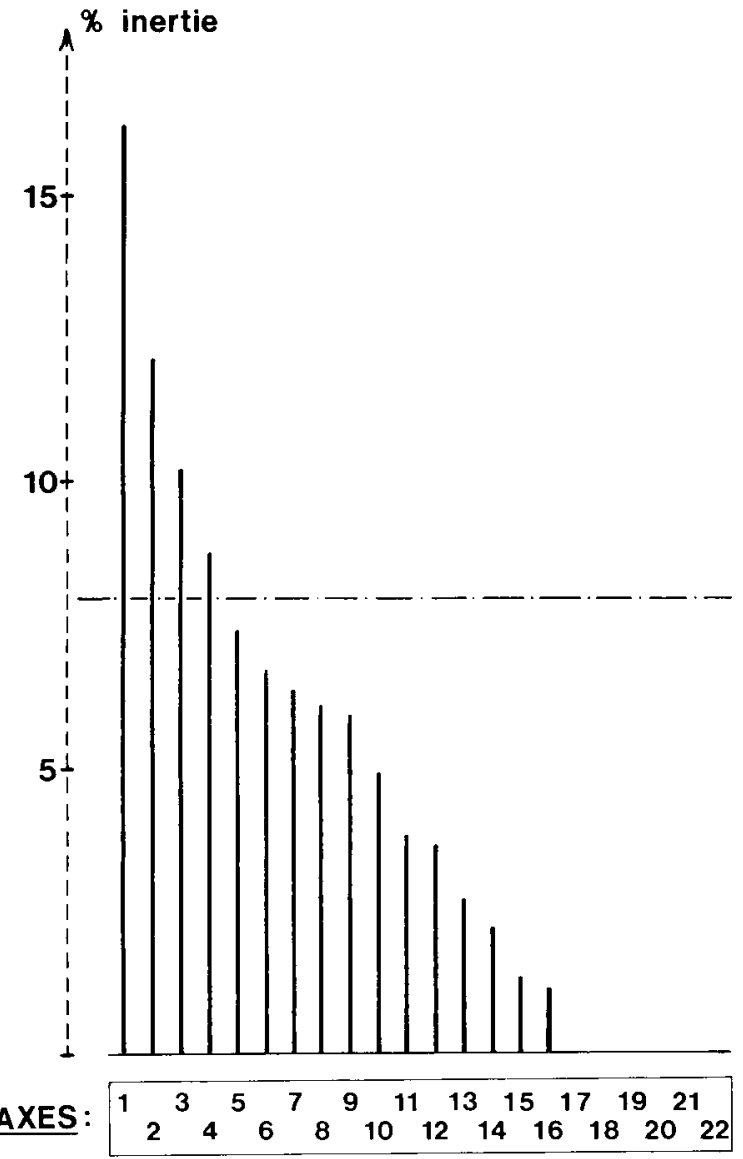

Figure 1

Histogramme des pourcentages d'inertie représentée par les axes factoriels de l'analyse des correspondances.

Histogram of the inertia percentages of factorial axes of correspondance analysis.

Le plan des 2 premiers axes (fig. 2) visualise la relation très étroite entre les 2 relevés de développement foliaire effectués à 13 jours d'intervalle ( $F 1$ et $F 2$ ) ainsi que les rapprochements fort diamètre du tronc-grande longueur maximale des rameaux (DT 4, LM 3), d'une part, et faible nombre de rameaux de l'année-fort degré de ramification (NR 1, RA 3), d'autre part. Longueur maximale des rameaux, diamètre du tronc et degré de ramification (LM, DT, RA) évoluent par ailleurs de façon assez simultanée.

L'axe 3 (10 p. 100 de l'information), contrairement aux 2 précédents, met en relief des modalités intermédiaires sur sa partie positive (les classes F 12, F 23, NR 2 constituent 24 p. 100 de l'axe et RA 2, 22,4 p. 100 à elle seule). Il leur oppose la précocité du feuillage (F 13, F 24) et une nouvelle manifestation de l'association faible nombre de rameauxfort degré de ramification (NR 1, RA 3). Ces 8 modalités déterminent 79 p. 100 de l'axe.

L'association des axes 1 et 3 (fig. 3) représente une structure typique en croissant de variables évoluant simultanément, à l'exception de la longueur du rameau le plus long, pour les modalités fortes (LM 2 et LM 3 ) et surtout du jaunissement du feuillage.

L'axe 4 (9 p. 100 de l'information) oppose des arbres de faible diamètre, à rameaux nombreux et précocité moyenne (DT 1, NR 3, F 12, F 23) à des individus à feuillage très précoce, diamètre assez élevé, rameaux très longs et en nombre moyen (F 13, F 24, DT 3, LM 3, NR 2). Ces modalités déterminent 80 p. 100 de l'axe.

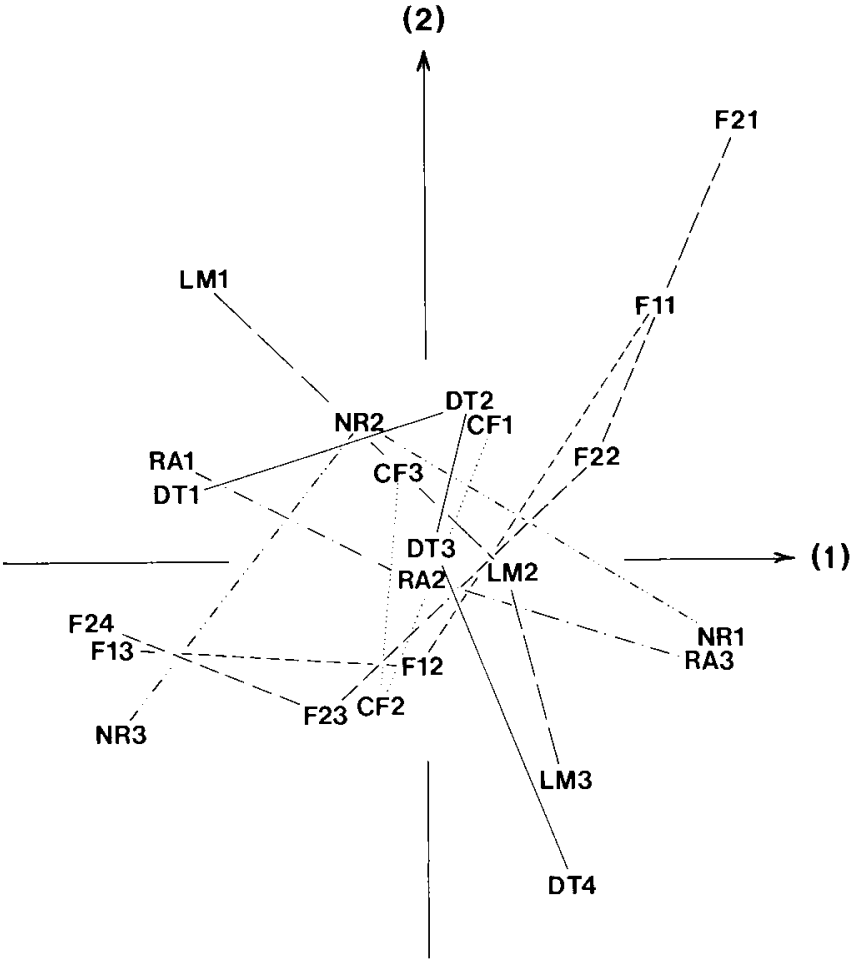

Figure 2

Plan des 2 premiers axes factoriels de l'analyse des correspondances. Plan of the first two axes of correspondance analysis.

(3)

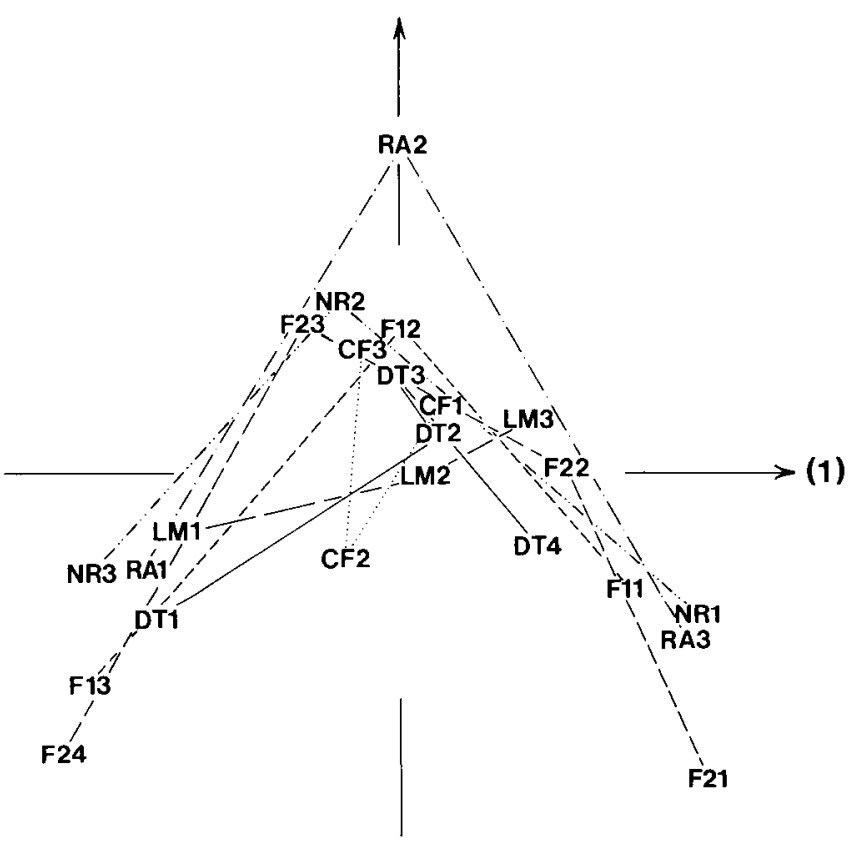

Figure 3

Plan des axes factoriels 1 et 3 de l'analyse des correspondances. Plan of axes 1 and 3 of correspondance analysis.

\section{B. Classification ascendante hiérarchique}

L'analyse factorielle des correspondances structure les modalités des variables phénologiques mais aussi les individus (arbres). Le nuage de ces 125 points ne permet pourtant pas de distinguer des groupes bien séparés les uns des autres. La prise en compte de 4 axes simultanément rendrait 


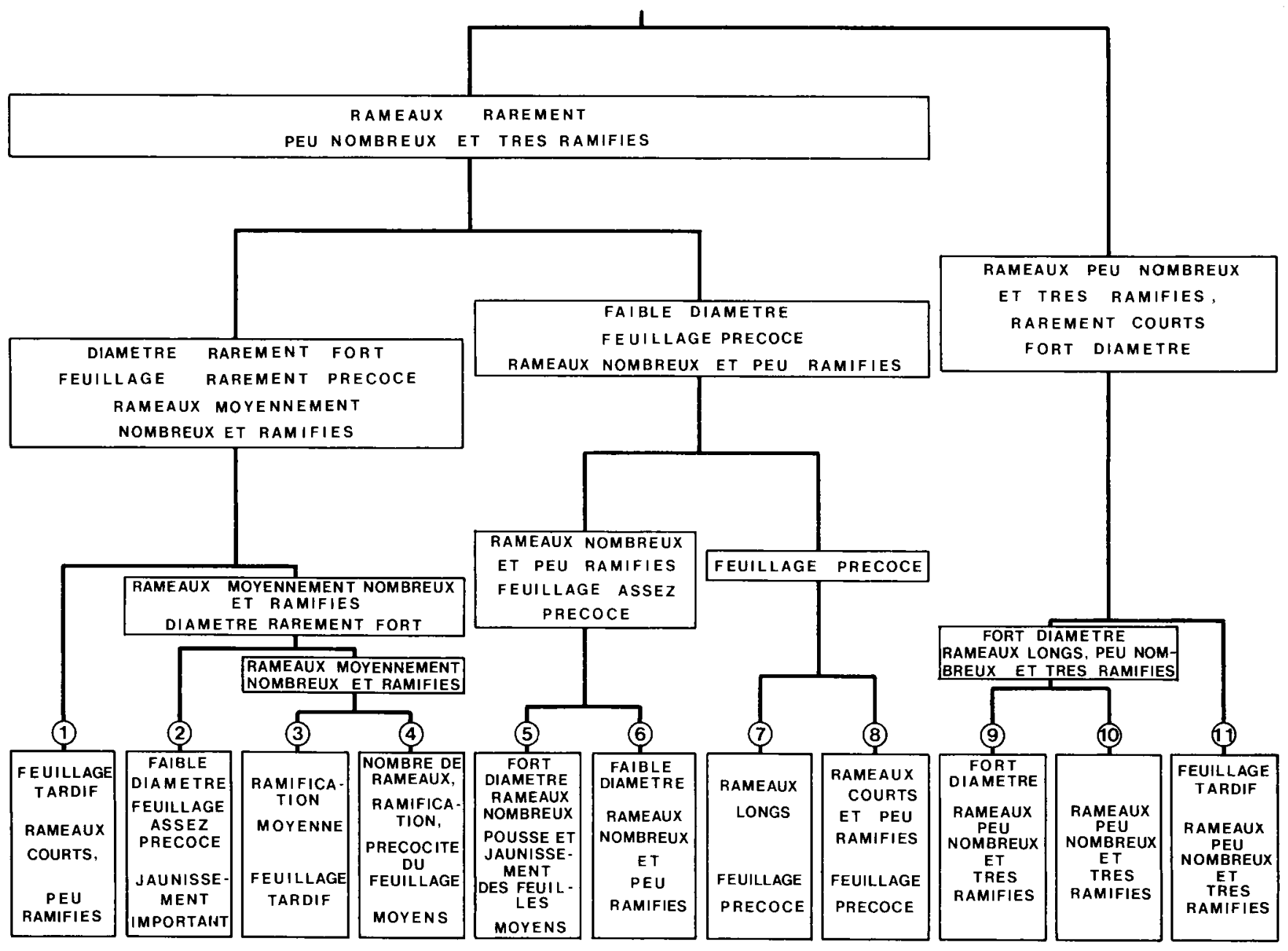

Figure 4

Arbre issu de la classification ascendante hiérarchique: les associations significatives des 2 branches issues de chaque noeud avec les modalités phénologiques sont figurées.

Hierarchical tree from cluster analysis : significative association between branches of each nodus and phenological data are figured.

d'ailleurs la détermination de ces groupes délicate. On utilise alors une classification ascendante hićrarchique, basée sur le critère de la maximisation du moment centré d'ordre 2 (minimisation de l'inertie intra-classes); clle agglomère progressivement les individus, puis les groupes, pour constituer un arbre hiérarchique (fig. 4). La classification est effectuée à partir des coordonnées sur les 4 premiers axes factoriels plutôt que sur les données initiales. Cette méthode permet de ne conserver que la part d'information qui contribue le plus à la structure d'ensemble, le «bruit de fond» étant alors rejeté sur les axes ultérieurs (dans cet exemple, les classifications ascendantes hiérarchiques réalisées avec un nombre supérieur d'axes produisent des hiérarchies de moins en moins nettes).

Afin de faciliter le choix d'un niveau de coupure de cette hiérarchie, il est possible d'utiliser la méthode suivante : en descendant l'arbre hiérarchique, au niveau de chaque nœud, on calcule la longueur moyenne de l'ensemble des branches inférieures, jusqu'aux nœuds les plus proches (« indice de coupure »). La figure 5A illustre les longueurs prises en compte dans ce calcul pour des niveaux de coupure définissant 3 groupes (coupure 1) ou 6 groupes (coupure 2). L'histogramme des valeurs de cet indice est représenté par la figure 5B. La diminution de l'indice de coupure est forte entre 3 et 4 groupes: l'on pourrait donc conserver la coupure du niveau 1. La chute de l'indice est encore forte pour 5 groupes et l'on pourrait également choisir ce niveau.
Il n'y a par contre aucune raison de conserver la coupure du niveau 2. On observe par ailleurs, en poursuivant le découpage, que l'indice se stabilise sensiblement jusqu'à la formation de 11 classes, pour chuter brutalement à 12 puis se stabiliser de nouveau. L'arbre hiérarchique a donc été finalement coupé à ce niveau.

Les caractéristiques phénologiques des groupes d'individus déterminés par un niveau de coupure donné peuvent être dégagées par les indices d'association et de signification de BORDET \& ALBUISSON (1974). Les principales caractéristiques des coupures successives sont représentées par la figure 4. Le tableau 2 présente les indices d'association et de signification des 11 groupes définitifs, en fonction des modalités initiales.

\section{Détermination des arbres échantillons}

Les arbres des différents groupes ne présentent pas de localisation particulière à l'intérieur du verger (fig. 6). Afin que les groupes à effectif faible soient suffisamment représentés, un nombre constant d'arbres a été retenu pour chacun. Une seconde stratification a alors été effectuée suivant une double contrainte : un effectif de 5 arbres par groupe et une répartition aussi uniforme que possible des individus (fig. 6). Cette répartition uniforme doit permettre 


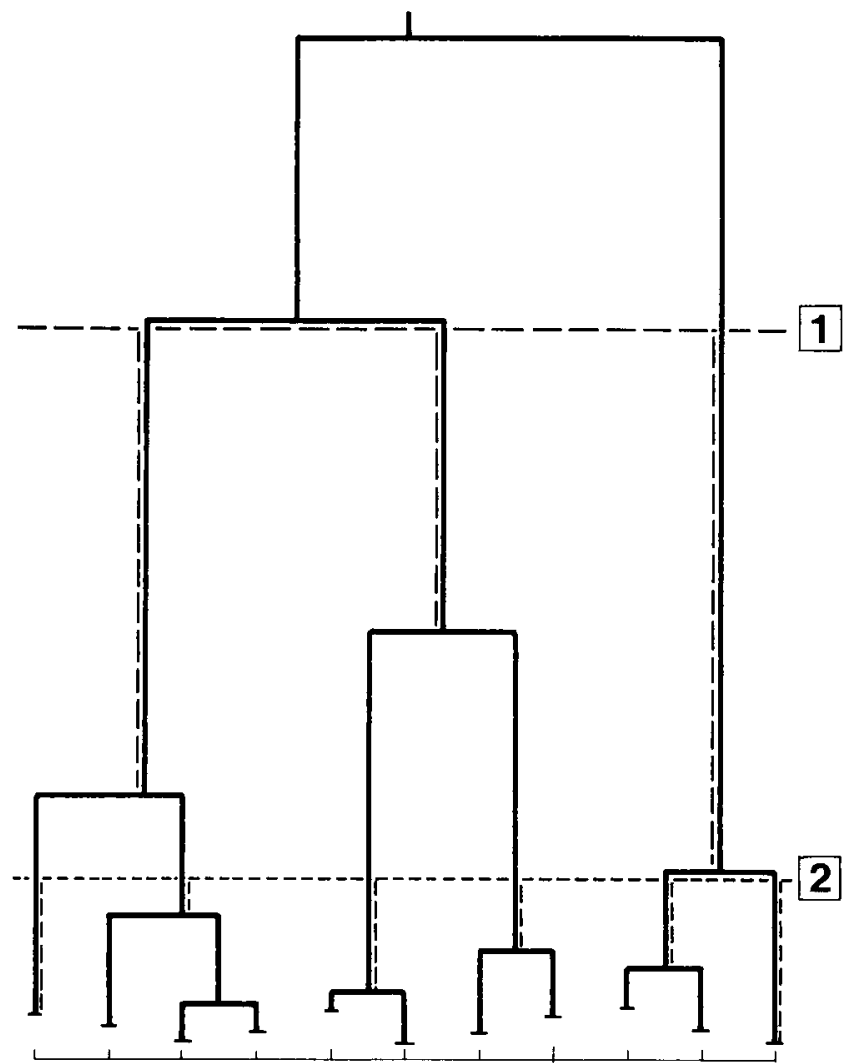

\begin{tabular}{|lllllllllll|}
1 & 2 & 3 & 4 & 5 & 6 & 7 & 8 & 9 & 10 & 11 \\
\hline
\end{tabular}

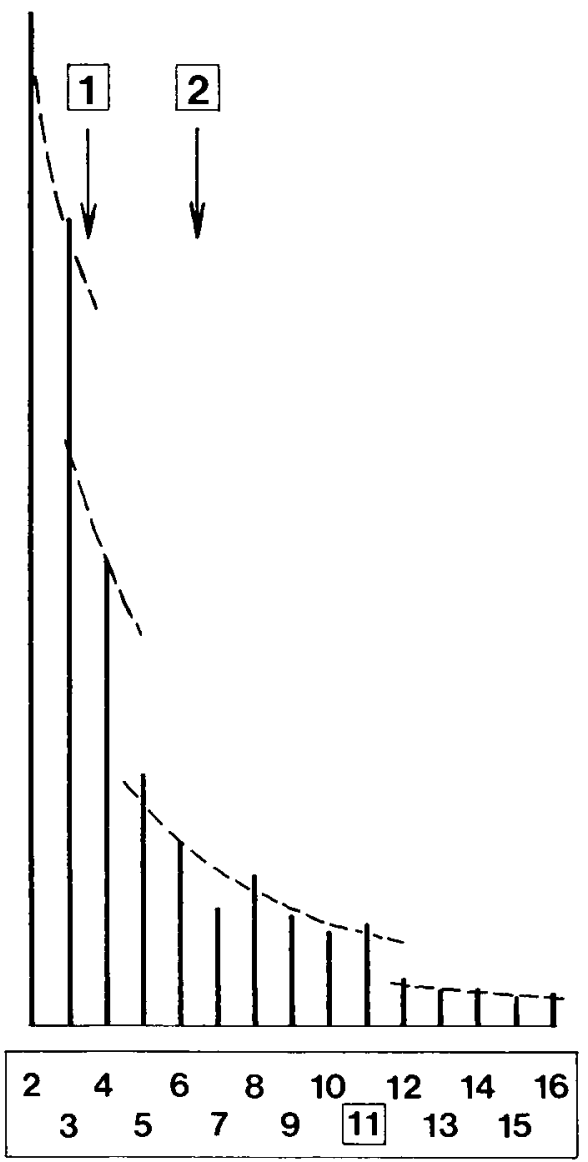

B)

Figure 5

Illustration du calcul de "l'indice de coupure 》:

A. - Représentation des longueurs mesurées pour le calcul de cet indice à 2 niveaux.

B. - Histogramme de l'indice de coupure pour des niveaux définissant de 2 à 16 groupes.

Computing of an index to help in determination of the hierarchical tree cutting level:

A. - Lengths used in the computing at 2 cutting levels.

$B$. - Histogram of the cutting index for levels giving 2 to 16 groups.

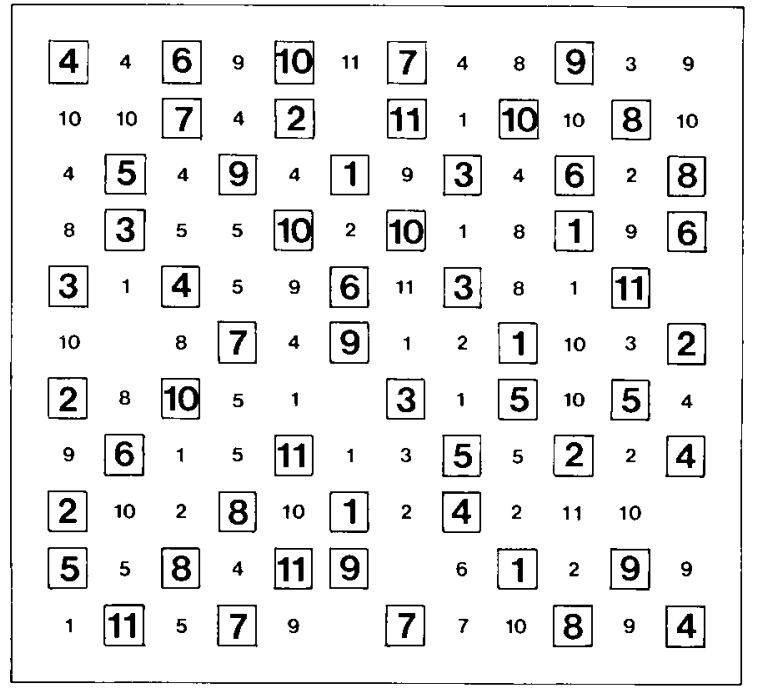

Figure 6

Répartition des arbres des différents groupes dans le verger et distribution des individus retenus pour l'échantillonnage.

Distribution of trees of the different groups in the orchard. The sampling trees are framed. de mettre en évidence des effets de lisières ou des gradients éventuels dans le verger.

\section{DISCUSSION ET CONCLUSION}

L'importance des caractéristiques phénologiques pour la définition de la "personnalité " d'un arbre a été rappelée par KolbE (1979). La prise en compte de variables de ce type est toutefois rare, à l'exception de travaux portant sur la croissance ou la productivité d'essences forestières (DAGNelie et al., 1978 ; THILl \& GRAYET, 1978 ; THILl \& PALM, 1979 ; WEST, 1980). Des études ont cependant été menées sur le développement végétatif de variétés de pêchers en fonction de la chute des feuilles de la saison précédente (COUVILLON \& LLOYD, 1978), de la thermopériode (COUVILlon \& HENDERSHOTT, 1974 ; EREZ et al., $1979 \mathrm{a}$ et b), de la concentration en oxygène (EREZ et al., 1980), etc.

Le choix des variables phénologiques utilisées dans cette étude a été dicté par des contraintes pratiques concernant notamment la durée des observations. Si les caractéristiques prises en compte sont suffisantes pour établir une stratification efficace de l'échantillonnage, un élément a été ajouté pour les travaux poursuivis actuellement sur ce verger : il s'agit de la répartition des fréquences des différents types de 
TABLEAU 2

Indices d'association ( $1^{r e}$ ligne) et de signification ( $2^{e}$ ligne) entre les groupes d'arbres issus de la classification ascendante hiérarchique et les modalités des différentes variables phénologiques

Association index (1st line) and signification index (2nd line) between the groups of trees obtained with cluster analysis and the classes of the different phenological data

\begin{tabular}{|c|c|c|c|c|c|c|c|c|c|c|c|}
\hline Groupes & 1 & 2 & 3 & 4 & 5 & 6 & 7 & 8 & 9 & 10 & 11 \\
\hline DT1 & & + & -- & -- & - & $\begin{array}{l}++++ \\
++++\end{array}$ & & ++ & & -- & \\
\hline DT2 & ++ & + & & ++ & -- & -- & - & & -- & + & ++ \\
\hline DT3 & & & ++ & ++ & & -- & +++ & & -- & & - \\
\hline DT4 & -- & -- & & -- & $\begin{array}{l}+++ \\
+++\end{array}$ & -- & -- & -- & $\begin{array}{c}+++ \\
++++\end{array}$ & + & -- \\
\hline LM1 & $\begin{array}{c}+++ \\
++++\end{array}$ & ++ & - & -- & -- & ++ & -- & $\begin{array}{c}++ \\
++\end{array}$ & -- & -- & - \\
\hline LM2 & -- & & +++ & & ++ & & --- & -- & & - & +++ \\
\hline LM3 & -- & -- & -- & ++ & & -- & $\begin{array}{c}+++ \\
+\end{array}$ & -- & ++ & ++ & -- \\
\hline NR1 & -- & -- & -- & -- & -- & -- & -- & -- & $\begin{array}{l}++++ \\
++++\end{array}$ & $\begin{array}{l}+++ \\
++++\end{array}$ & $\begin{array}{c}+++ \\
+++\end{array}$ \\
\hline NR2 & $++t$ & +++ & ++++ & $\begin{array}{c}++++ \\
+++\end{array}$ & --- & $-\cdots--$ & +++ & + & --- & ---- & ---- \\
\hline NR3 & - & & -- & -- & $\begin{array}{l}++++ \\
++++\end{array}$ & $\begin{array}{l}++++ \\
++++\end{array}$ & & + & -- & -- & -- \\
\hline RA1 & $\begin{array}{l}+++ \\
++++\end{array}$ & ++ & --- & --- & +++ & $\begin{array}{c}+++ \\
+\end{array}$ & --- & $\begin{array}{c}+++ \\
+++\end{array}$ & --- & --- & --- \\
\hline RA2 & -- & + & $\begin{array}{l}++++ \\
++++\end{array}$ & $\begin{array}{l}++++ \\
++++\end{array}$ & & -- & ++ & -- & -- & -- & -- \\
\hline RA3 & -- & --- & --- & -- & --- & --- & ++ & --- & $\begin{array}{l}++++ \\
++++\end{array}$ & $\begin{array}{l}++++ \\
++++\end{array}$ & $\begin{array}{c}+++ \\
+++\end{array}$ \\
\hline F11 & $\begin{array}{l}+++ \\
+++\end{array}$ & -- & $\begin{array}{l}+++ \\
++++\end{array}$ & -- & -- & -- & -- & -- & -- & ++ & $\begin{array}{l}++++ \\
++++\end{array}$ \\
\hline F12 & --- & +++ & ---- & $\begin{array}{c}+++ \\
+\end{array}$ & ++ & ++ & --- & ---- & ++++ & -- & ---- \\
\hline F13 & -- & & -- & -- & + & + & $\begin{array}{c}+++ \\
+++\end{array}$ & $\begin{array}{l}++++ \\
++++\end{array}$ & - & - & -- \\
\hline F21 & + & - & & - & - & & & & - & & $\begin{array}{c}++ \\
+++t\end{array}$ \\
\hline F22 & $\begin{array}{c}+t \\
+\end{array}$ & -- & ++++ & & --- & -- & --- & --- & + & +++ & + \\
\hline F23 & --- & $\begin{array}{c}++ \\
+\end{array}$ & --- & ++ & $\begin{array}{c}+++ \\
++\end{array}$ & +++ & --- & --- & + & $-\cdots$ & --- \\
\hline F24 & - & - & - & - & & - & $\begin{array}{l}++++ \\
++++\end{array}$ & $\begin{array}{l}++++ \\
++++\end{array}$ & - & & - \\
\hline $\mathrm{CF} 1$ & +++ & - & & ++ & ---- & ++ & --- & -- & ++ & --- & +++ \\
\hline $\mathrm{CF} 2$ & -- & - & - & -- & $\begin{array}{c}+++ \\
+\end{array}$ & -- & ++ & ++ & - & ++ & --- \\
\hline $\mathrm{CF} 3$ & - & $\begin{array}{c}++ \\
+\end{array}$ & & - & - & & + & & - & & \\
\hline
\end{tabular}

bourgeons. L'importance de cette variable descriptive a été soulignée par plusieurs auteurs, en relation avec les autres paramètres phénologiques (KOLBE, 1979), la productivité du pommier (FoURCADE, 1979), les caractéristiques variétales de plusieurs espèces fruitières (VITKOVSKII, 1978) ou les porte-greffes du pêcher (EDIN, 1979).

La mise en parallèle de variables phénologiques avec les populations de ravageurs, hormis la description des formes caractéristiques de dégâts, est peu développée: NAGY
(1978) a suivi l'évolution de perforations durant la croissance des feuilles de plusieurs espèces forestières ; TAKEDA (1979 a et b) a observé la microrépartition de différents pucerons pendant la poussée végétative de pommiers.

L'échantillonnage des populations animales à l'intéricur de l'arbre a retenu l'attention de plusieurs auteurs tels que LECLANT (1978) pour les pucerons du pêcher, FAUVEL et al. (1978) pour les œufs d'hiver de l'acarien rouge du pommier, ou CARrero (1979) pour l'aleurode des agrumes. Pour le 
choix des arbres à échantillonner, l'on se contente le plus souvent des méthodes classiques rappelées par DAGNELIE $\boldsymbol{e t}$ al. (1978), tirage au hasard ou répartition uniforme, parfois complétées par une stratification sommaire, effectuée $a$ priori.

L'analyse factorielle des correspondances permet, grâce au codage en classes, d'exploiter des données simplifiées, par exemple sous forme d'indices; une étude préliminaire du milieu est ainsi réalisable sans trop d'investissement en temps. La typologie automatique, appliquée aux résultats de cette analyse, est alors particulièrement appropriée pour la stratification des individus en vue d'un échantillonnage plus précis.

Reçu le 16 mars 1981. Accepté le 27 avril 1981.

\section{RÉFÉRENCES BIBLIOGRAPHIQUES}

Bordet J. P., Albuisson M., 1974. Méthodes de dépouillement de tableaux croisés. Note interne, ARLAB, 25, $56 \mathrm{p}$.

Carrero J. M., 1979. Contribucion al estudio de la biologia de la « mosca blanca » de los agrios, Aleurothrixus floccosus Mask., en la region valenciana. V. Estudios previos al establecimiento de la dinamica poblacional. 2. Distribucion de brotaciones. An. INIA, Ser. Prot. Veg., 9: 177-181.

Couvillon G. A., Hendershott C. H., 1974. A characterization of the "after-rest " period of flower buds of two peach cultivars of different chilling requirements. J. amer. Soc. Hortic. Sci., 99 : 2326.

Couvillon G. A., Lloyd D. A., 1978. Summer defoliation effects on peach spring bud development. Hortscience, 13 : 53-54.

Dagnelie P., Grayet J. P., Rondeux J., Thill A., 1978. Estimation de la production forestière : principes et applications. Bull. Soc. roy. For. de Belg., 85 : 97-122.

Edin M., 1979. Porte-greffe du pêcher. Etude de son influence sur certains aspects du comportement de la variété greffée. CTIFLDocuments, $3^{e}$ Trim. 1979, 63 : 61-71.

Erez A., Couvillon G. A., Hendershott C. H., 1979 a. Quantitative chilling enhancement and negation in peach buds by high temperatures in a daily cycle. J. amer. Soc. Hortic. Sci., 104: 536-540.

Erez A., Couvillon G. A., Hendershott C. H., 1979 b. The effect of cycle length on chilling negation by high temperatures in dormant peach leaf buds. J. amer. Soc. Hortic. Sci., 104: 573-576.

Erez A., Couvillon G. A., Kays S. J., 1980. The effect of oxygen concentration on the release of peach leaf buds from rest. Hortscience, $15: 39-41$.

Fauvel G., Audemard H., Rodolphe F., Rambier A., 1978. Le recensement des oufs d'hiver de l'acarien rouge, Panonychus ulmi Koch sur le pommier. I. Comparaison des méthodes actuelles et remarques sur l'influence de quelques facteurs dans leur variabilité. Ann. Zool. Ecol. anim., 10:461-482.
Fourcade P., 1979. Répercussions physiologiques de l'irrigation fertilisante par goutte à goutte. Purpan, 111: 99-141.

Jambu M., 1978. Classification automatique pour l'analyse des données. 1. Méthodes et algorithmes. Edit. DUNOD, Paris, $310 \mathrm{p}$. Jambu M., Lebeaux M.-O., 1978. Classification automatique pour l'analyse des données. 2. Logiciels. Édit. DUNOD, Paris, 399 p.

Kolbe W., 1979. Jahreszeitlicher Verlauf der Entwicklungsstadien bei Obstarten in Beziehung zu Jahreswitterung und Pflanzenschutzmassnahmen. Pflanzenschutz-Nachrichten Bayer, 32 : 97-163.

Leclant F., 1978. Etude bioécologique des aphides de la région méditerranéenne. Implications agronomiques. Thèse Doc. ès Sci., Univ, de Montpellier, $318 \mathrm{p}$.

Nagy M., 1978. Experimental analysis of the connection between leaf growth and insect consumption. Acta Bot. Acad. Sci. Hung., 24 : 307-326.

Takeda S., 1979 a. Spatial distribution of the apple leaf-curling aphid, Myzus malisuctus Matsumura, and the spirea aphid, Aphis spiraecola Patch, on apple seedlings. Appl. Ent. Zool., 14 : 356359.

Takeda S., $1979 \mathrm{~b}$. Movement of adults and larve of the apple leafcurling aphid Myzus malisuctus Matsumura, and the spirea aphid Aphis spiraecola Patch, on the apple tree. Appl. Ent. Zool., 14 : $487-490$

Thill A., Grayet J. P., 1978. Etude dendrométrique du hêtre commun. Note technique du Centre d'Écologie forestière et rurale de Gembloux, 32: $60 \mathrm{p}$.

Thill A., Palm R., 1979. Etude dendrométrique des chênes indigènes. Note technique du Centre d'Écologie forestière et rurale de Gembloux, 33 : $54 \mathrm{p}$.

Vitkovskii V. L., 1978. Principles governing the formation of vegetative and reproductive organs in fruit plants. Tr. Prikl. Bot. genet. Sel., $62: 59-78$.

West P. W., 1980. Use of diameter increment and basal area increment in tree growth studies. Can. J. For. Res., 10:71-77. 